\title{
Postero-apical thoracic schwannoma with cervical extension resected by complete video-assisted thoracoscopic surgery
}

\author{
Harry Etienne, Apostolos C. Agrafiotis, Hicham Masmoudi, Jalal Assouad \\ Department of Thoracic Surgery, Tenon University Hospital, Paris, France
}

\begin{abstract}
Schwannomas or neurilemmomas are benign tumors developed from the peripheral nervous system. Complete video-assisted thoracic surgery (cVATS) has set itself over the years as the preferred approach for the removal of small mediastinal neurogenic tumors. However, in case of apical location, complete VATS seems challenging because of proximity with the subclavian artery and/or elements of the brachial plexus. In case of a cVATS procedure, some authors prefer enucleation instead of resection, with a higher risk of relapse. We present two cases of cVATS resection of thoracic apical schwannomas.
\end{abstract}

\section{Introduction}

Schwannomas or neurilemmomas are benign peripheral tumors developed from nerve sheaths [1]. In the thoracic apical location, this tumor can cause vascular and/or neurological symptoms by compression of the subclavian vessels and/or elements of the brachial plexus [1].

Video-assisted thoracic surgery (VATS) is the preferred tech-

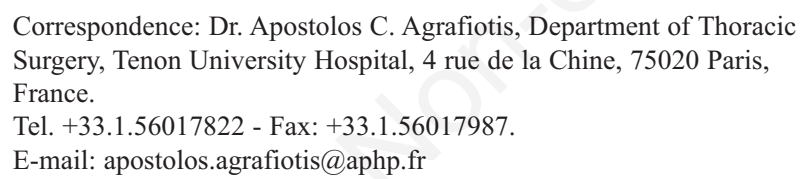

Keywords: Schwannoma; complete VATS; neurilemmoma; cervical extension.

Contributions: All the authors contributed equally.

Conflict of interest: The authors declare no conflict of interest.

Received for publication: 25 March 2019.

Accepted for publication: 15 May 209.

(C) Copyright H. Etienne et al., 2019

Licensee PAGEPress, Italy

Monaldi Archives for Chest Disease 2019; 89:1073

doi: 10.4081/monaldi.2019.1073

This article is distributed under the terms of the Creative Commons Attribution Noncommercial License (by-nc 4.0) which permits any noncommercial use, distribution, and reproduction in any medium, provided the original author(s) and source are credited. nique for management of small mediastinal neurogenic tumor without intraforaminal extension [2]. However, in apical location, either resection by VATS combined with a supraclavicular incision or enucleation by VATS seem to be currently the most frequent approaches because of the proximity to the vascular and neurological structures. We present here two cases of thoracic apical schwannomas that were resected exclusively by complete VATS (cVATS).

\section{Cases Report}

\section{Case \#1}

A 57-year-old female patient with a history of smoking was admitted to the Department of Thoracic Surgery for a left apical opacity discovered on a chest X-ray because of a persistent cough. No specific signs, including neurologic or vascular symptoms, were found during clinical examination. Computed tomography (CT) scan described a $48 \times 35 \times 46 \mathrm{~mm}$ apical left thoracic mass, in proximity with the left subclavian artery. The magnetic resonance imaging (MRI) showed a heterogeneous signal enhanced by gadolinium's injection, suggestive of a neurilemmoma (Figure 1). Preoperative assessment included a bronchoscopy and respiratory function tests that were within normal limits. Complete VATS resection was chosen as the preferred approach.

Surgical technique: under general anesthesia, in right lateral position, three incisions were performed: a utility incision of $2 \mathrm{~cm}$ on the superior border of the $4^{\text {th }}$ rib, in the mid-axillary line and two additional incisions to achieve triangulation. The tumor seemed to be developed from the left sympathetic chain. It was encapsulated. The mediastinal pleura surrounding the tumor was first opened. The tumor was then dissected and separated from the left subclavian artery using cautery and endo-peanuts (Figure 2). After the tumor extraction, a chest tube (24 Fr) was left in place for drainage.

Post-operative course was uneventful. The chest tube was removed two days after surgery and the patient was discharged the day after. One month after surgery, the patient was totally asymptomatic. The chest X-ray was normal. Final histological analysis confirmed the diagnosis of a benign schwannoma.

\section{Case \#2}

A 39-year-old female patient with a history of asthma was referred to the Department of Thoracic Surgery for the treatment of a left postero-apical mass which was discovered during a clinical workup for persisting cough. CT scan showed a close relationship with the spine and the left subclavian vessels. The MRI excluded intraforaminal extension (Figure 3). Overall presentation 
was highly suggestive of a schwannoma. Preoperative functional workup was within normal limits.

Surgical approach was the same as for our first patient. The chest tube was removed on post-operative day 2 and the patient was discharged the day after. Post-operative control in the outpatient clinic one month later did not reveal any anomaly. The chest $\mathrm{X}$-ray was normal. Histopathological analysis confirmed the initial hypothesis of a benign schwannoma. Both patients will be examined at the outpatient clinic once per year with a chest CT scan.

\section{Discussion}

Complete VATS is an interesting and secure approach for mediastinal neurogenic tumors without intraforaminal extension. Its benefits include shorter operative time, fewer blood loss, shorter hospital stay and decreased consumption of analgesics [3]. It appears as the procedure of choice for small mediastinal neurogenic tumors [2]. Thoracotomy is generally reserved for larger tumors and in case of suspicion for malignancy [2].

However, surgical strategy for thoracic apical neurilemmomas favors resection by a posterolateral thoracotomy, a supraclavicular incision, a combined VATS and supraclavicular incision or enucleation by cVATS [1]. Enucleation by cVATS exposes to a risk of relapse and seems inappropriate [4]. Neurogenic mediastinal tumors arising from intercostals nerves can be exclusively intrathoracic or they may have an intraforaminal expansion, as well (hourglass or dumbbell tumors) [5-7]. In case of dumbbell tumors a combined access is necessary through thoracotomy or thoracoscopy and laminectomy. The MRI is the imaging modality of choice in order to detect an intraforaminal extension [5]. Resection of apical thoracic neurilemmomas by complete minimally invasive approach appears challenging because of close proximity with subclavian artery and brachial plexus. Finley et al., reported the case of a 63-year-old patient who underwent a robotically-assisted thoracoscopic resection of a paraspinal apical schwannoma [8]. With the two cases presented herein, we showed that it is also safe and feasible to resect an api- cal thoracic schwannoma with cVATS. There are case series in the literature concerning the feasibility and safety of this approach. In the series of Chen et al., 74 out of 121 posterior mediastinal neurogenic tumors were resected by VATS [7]. However, conversion to thoracotomy should be performed in case of technical difficulties, the risk of incomplete resection or the possibility of malignancy. Complete resection is important, even for benign tumors, as there is the risk of local recurrence $[5,6]$. As far as the postoperative follow-up is concerned, during the first postoperative outpatient visit a simple chest X-ray is usually sufficient. However, a longer follow-up with a chest CT scan must be implemented in order to detect tumor recurrence or other similar lesions.

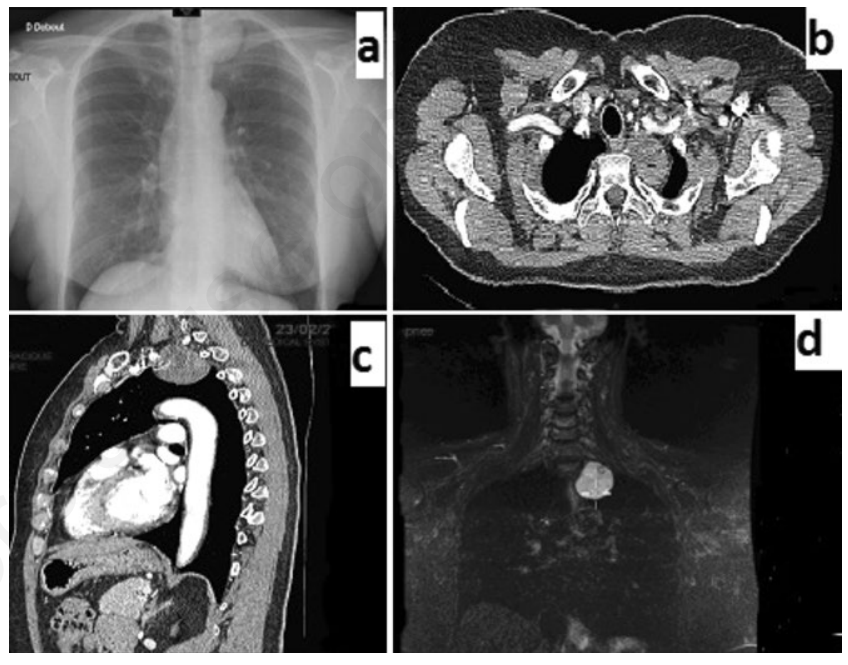

Figure 1. Case \#1. a) Chest X-Ray showing a left apical mass; b,c) CT scan showing the tumor in close contact with the left subclavian artery; d) MRI showing a heterogeneous mass compatible with a neurilemmoma.
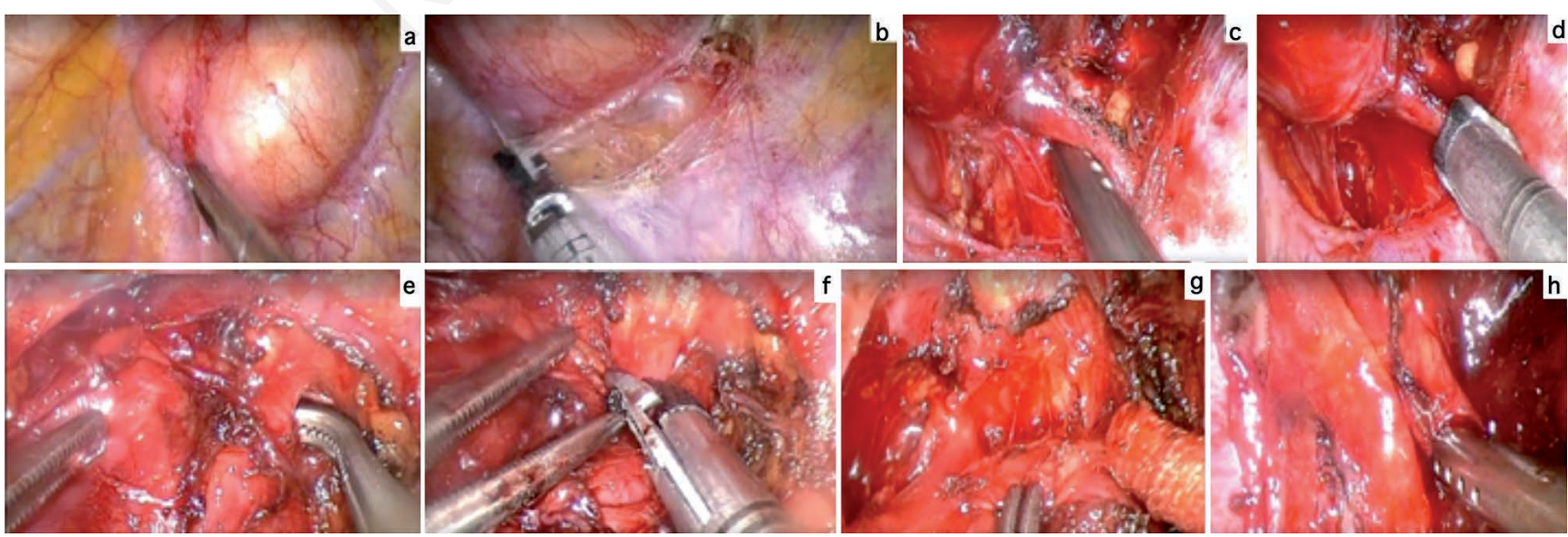

Figure 2. Case \# 1. Intraoperative images showing: a) the lesion; b) incision of the parietal pleural around the tumor; c) dissection of the nerve distally; d) clipping of the nerve distally; e) dissection of the nerve proximally; f) clipping of the nerve proximally; g) dissection of the upper pole of the tumor; $h$ ) dissection near the left subclavian artery. 

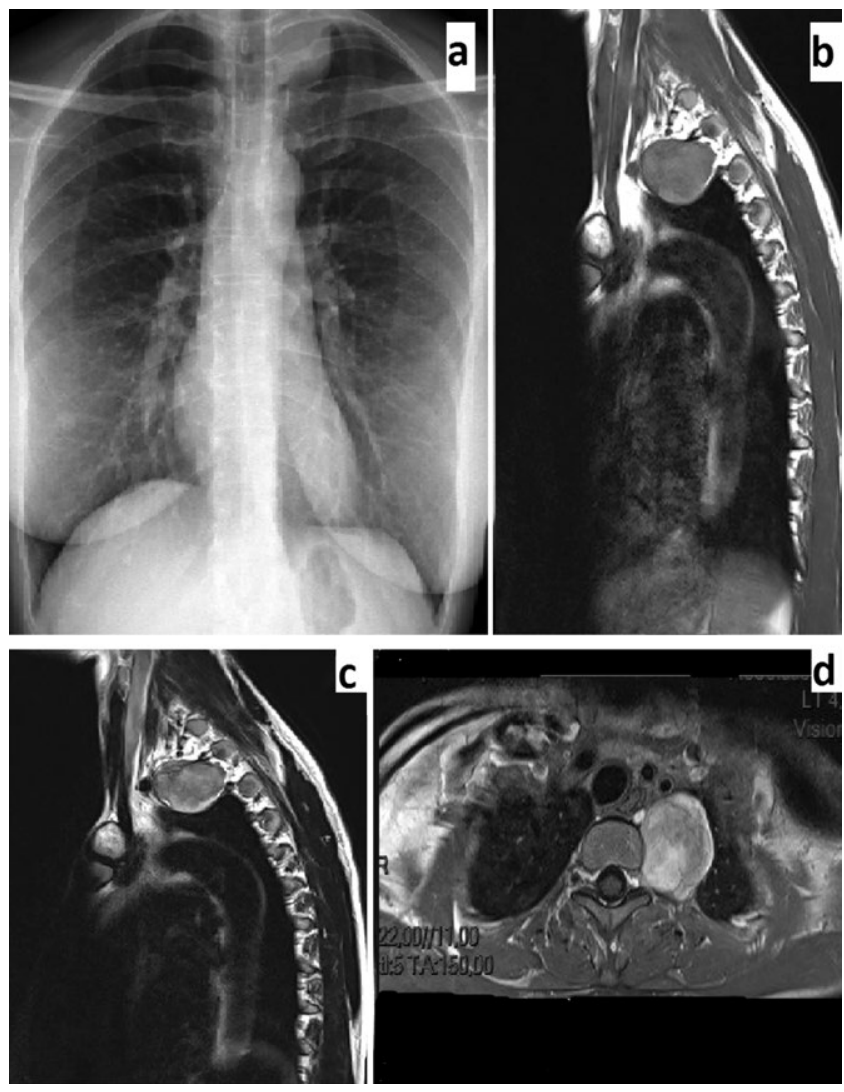

Figure 3. Case \#2. a) Chest X-ray; b,c,d) MRI images showing the apical lesion.

\section{Conclusions}

Neurilemmomas are benign neurogenic tumors with excellent prognosis after surgical resection. In apical location, removing these tumors by complete VATS is a feasible and safe option. Complete pre-operative assessment is warranted in order to choose the best surgical approach. MRI is a very useful tool to evaluate the relationship between the tumor and neurovascular structures. A longer follow-up after surgery is advised in order to detect tumor recurrence or other similar lesions.

\section{References}

1. Endo S, Murayama F, Otani S, et al. Alternative surgical approaches for apical neurinomas: a thoracoscopic approach. Ann Thorac Surg 2005;80:295-8.

2. Li Y, Wang J. Experience of video-assisted thoracoscopic resection for posterior mediastinal neurogenic tumours: a retrospective analysis of 58 patients. ANZ J Surg 2013;83:664-8.

3. Cansever L, Kocaturk CI, Cinar HU, et al. Benign posterior mediastinal neurogenic tumors: results of a comparative study into video-assisted thoracic surgery and thoracotomy (13 years' experience). Thorac Cardiovasc Surg 2010;58:473-5.

4. Illuminati G, Pizzardi G, Minni A, et al. Superiority of resection over enucleation for schwannomas of the cervical vagus nerve: A retrospective cohort study of 22 consecutive patients. Int J Surg 2016;29:74-8.

5. Okada D, Koizumi K, Haraguchi S, et al. A case of dumbbell tumor of the superior mediastinum removed by combined thoracoscopic surgery. J Nihon Med Sch 2002;69:58-61.

6. Nakamura H, Komagata M, Nishiyama M, et al. Resection of a dumbbell-shaped thoracic neurinoma by hemilaminectomy: a case report. Ann Thorac Cardiovasc Surg 2007;13:36-39.

7. Chen X, Ma Q, Wang S, et al. Surgical treatment of posterior mediastinal neurogenic tumors. J Surg Oncol 2019;119:807-13.

8. Finley D, Sherman JH, Avila E, et al. Thorascopic resection of an apical paraspinal schwannoma using the da Vinci surgical system. J Neurol Surg A Cent Eur Neurosurg 2014;75:58-63. 
